\title{
Recent results from the T2K experiment
}

\author{
M. Lawe ${ }^{1, \star}$ for the T2K Collaboration
}

${ }^{1}$ Lancaster University, Bailrigg, Lancaster, United Kingdom, LA1 4YW

\begin{abstract}
T2K is a long-baseline neutrino oscillation experiment based in Japan. It was the first experiment to observe $v_{e}$ appearance in a $v_{\mu}$ beam, and has more recently produced the highest precision measurement of the oscillation parameter $\theta_{23}$ and has begun to probe the charge-parity violation phase $\delta$. Using a $v_{\mu}$ beam disappearance sample, T2K measures $\sin ^{2} \theta_{23}=0.514_{-0.056}^{+0.055}$ and $\Delta m_{32}^{2}=(2.51 \pm 0.10) \times 10^{-3} \mathrm{eV}^{2} / \mathrm{c}^{4}$ assuming the normal mass hierarchy, and $\sin ^{2} \theta_{23}=0.511 \pm 0.055$ and $\Delta m_{13}^{2}=(2.48 \pm 0.10) \times 10^{-3} \mathrm{eV}^{2} / \mathrm{c}^{4}$ assuming the inverted mass hierarchy. A joint fit between $v_{\mu}$ beam appearance and disappearance samples produces consistent results for $\sin ^{2} \theta_{23}$ and $\Delta m_{32}^{2}$ whilst also excluding at $90 \%$ confidence level the regions $\delta=[0.15,0.83] \pi$ for the normal hierarchy and $\delta=[-0.08,1.09] \pi$. Using a $\bar{v}_{\mu}$ beam disappearance sample, $\sin ^{2} \bar{\theta}_{23}=0.45_{-0.12}^{+0.29}$ and $\left|\Delta \bar{m}_{32}^{2}\right|=(2.51 \pm 0.28) \times 10^{-3} \mathrm{eV}^{2} / \mathrm{c}^{4}$, consistent with previous measurements and the T2K $v_{\mu}$ sample.
\end{abstract}

\section{Neutrino Oscillation Theory}

The Pontecorvo-Maki-Nakagawa-Sakata (PMNS) formalism for neutrino oscillations describes the relation between the flavour eigenstates, $v_{\alpha}$, through which they interact, and the mass eigenstates, $v_{i}$, through which they propagate [1]

$$
\underbrace{\left(\begin{array}{c}
v_{e} \\
v_{\mu} \\
v_{\tau}
\end{array}\right)}_{\text {Flavour state }}=\underbrace{\left(\begin{array}{ccc}
U_{e 1} & U_{e 2} & U_{e 3} \\
U_{\mu 1} & U_{\mu 2} & U_{\mu 3} \\
U_{\tau 1} & U_{\tau 2} & U_{\tau 3}
\end{array}\right)}_{\text {Mixing matrix }} \underbrace{\left(\begin{array}{c}
v_{1} \\
v_{2} \\
v_{3}
\end{array}\right)}_{\text {Mass state }} .
$$

The PMNS mixing matrix, $U$, describes the $3 \mathrm{D}$ rotations between the mass and flavour eigenstates and is traditionally expressed as the product of three $2 \mathrm{D}$ rotation matrices

$$
U=\left(\begin{array}{ccc}
1 & 0 & 0 \\
0 & c_{23} & s_{23} \\
0 & -s_{23} & c_{23}
\end{array}\right)\left(\begin{array}{ccc}
c_{13} & 0 & s_{13} e^{-i \delta} \\
0 & 1 & 0 \\
-s_{13} e^{i \delta} & 0 & c_{13}
\end{array}\right)\left(\begin{array}{ccc}
c_{12} & s_{12} & 0 \\
-s_{12} & c_{12} & 0 \\
0 & 0 & 1
\end{array}\right),
$$

where $c_{i j} \equiv \cos \theta_{i j}$ and $s_{i j} \equiv \sin \theta_{i j}$, the cosine and sine of the three mixing angles, $\theta_{12}, \theta_{23}$ and $\theta_{13}$, and $\delta$ is the Dirac Charge-Parity (CP) violating phase.

^e-mail: m.lawe@lancaster.ac.uk 
The probability that a neutrino produced in flavour state $v_{\alpha}$, with energy $E$, is detected as flavour state $v_{\beta}$ after propagating a distance $L$ is given by

$$
P_{\alpha \rightarrow \beta}=\left|\left\langle v_{\alpha} \mid v_{\beta}(t)\right\rangle\right|^{2}=\left|\sum_{i j} U_{\alpha i}^{*} U_{\beta i} U_{\alpha j} U_{\beta j}^{*} e^{-i \frac{\Delta m_{i j}^{2}}{2} \frac{L}{E}}\right|,
$$

where $\Delta m_{i j}^{2}=m_{i}^{2}-m_{j}^{2}$, the mass squared difference between the mass eigenstates.

Three flavour neutrino oscillations are therefore dependent upon six measurable parameters, the three mixing angles $\theta_{12}, \theta_{23}$ and $\theta_{13}$, the CP-violating phase $\delta$, and two mass squared differences $\Delta m_{32}^{2}$ and $\Delta m_{21}^{2}$.

The dependence on $\frac{L}{E}$ in Equation 3 allows experiments to tune their baseline and neutrino energy to maximise the oscillation probability, and therefore their sensitivity to measurements of the oscillation parameters.

There are currently three open questions remaining within the PMNS framework,

- What is the sign of $\Delta m_{32}^{2}$ ? Is $\Delta m_{32}^{2}>0$, known as the "normal" neutrino mass ordering, or is $\Delta m_{32}^{2}<0$, known as the "inverted" neutrino mass ordering.

- What is the precise value of $\theta_{23}$ ? Is it maximal, $\theta_{23}=45^{\circ}$, or does it lie in the first, $\theta_{23}<45^{\circ}$, or second, $\theta_{23}>45^{\circ}$, octant?

- Is there Charge-Parity violation in the lepton sector? Requires $\sin \delta \neq 0$.

By measuring $v_{\mu}\left(\bar{v}_{\mu}\right)$-disappearance and $v_{e}\left(\bar{v}_{e}\right)$-appearance within an initially produced $v_{\mu}\left(\bar{v}_{\mu}\right)$ (anti)neutrino beam, the Tōkai-to-Kamioka (T2K) experiment can address these questions.

\section{The T2K Experiment}

The T2K experiment [2] is a long-baseline neutrino oscillation experiment based in Japan. The experiment produces a neutrino beam at the J-PARC research facility in Tōkai village on Japan's east coast. The beam properties are measured with a suite of near detectors at the J-PARC site, and $295 \mathrm{~km}$ away with the Super-Kamiokande (SK) detector in Kamioka. T2K was the first long-baseline neutrino oscillation experiment to use an off-axis design to tune the $\frac{L}{E}$ oscillation dependence to the oscillation maximum at SK.

\subsection{Beamline}

The T2K neutrino beam [3] is produced by impinging a $30 \mathrm{GeV}$ proton beam upon a graphite target. The interaction produces a large flux of mesons, predominately charged pions, which are charge selected and focused by a series of three magnetic horns. The mesons then enter a decay volume where they decay in-flight to produce neutrinos; the dominant process being $\pi^{+} \rightarrow \mu^{+}+v_{\mu}$. By reversing the polarity of the magnetic horns an antineutrino beam can be produced via $\pi^{-} \rightarrow \mu^{-}+\bar{v}_{\mu}$.

\subsection{Off-Axis Design}

The T2K neutrino beam centre is directed $2.5^{\circ}$ away from SK. Doing so tunes the beam profile into a narrowband neutrino flux spectrum, peaking at $0.6 \mathrm{GeV}$. This energy and baseline combination maximises the oscillation probability at SK, as seen in Figure 1, whilst reducing backgrounds. 


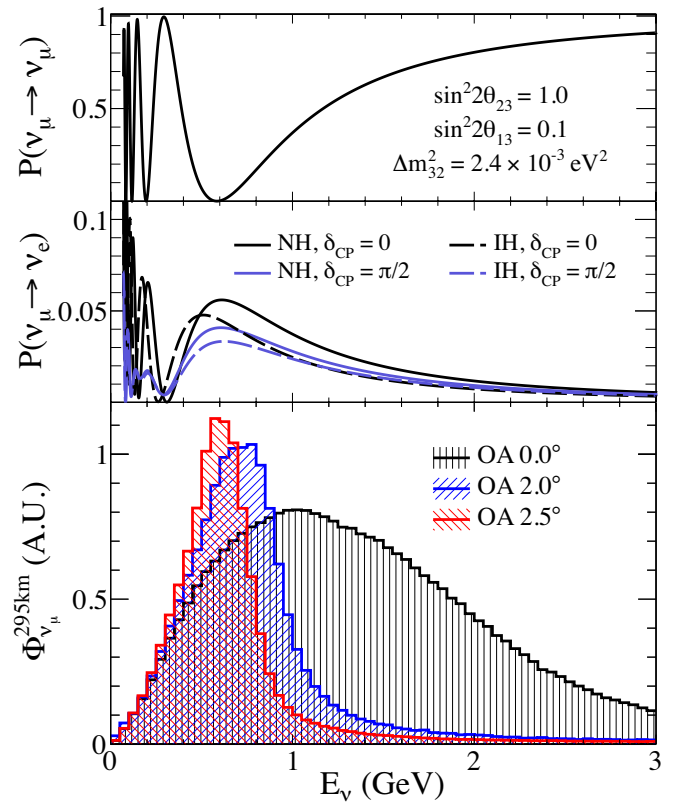

Figure 1. T2K neutrino flux spectrum for three different off-axis angles (lower panel), compared to $v_{\mu}$ survival probability (upper panel) and $v_{e}$ appearance probability (central panel) as a function of neutrino energy at the T2K baseline of $295 \mathrm{~km}$.

\subsection{Near Detectors}

T2K uses two near detectors, the on-axis Interactive Neutrino GRID (INGRID) and the off-axis Near Detector at $280 \mathrm{~m}$ (ND280). Both detectors are positioned within a pit $280 \mathrm{~m}$ downstream of the proton target at the J-PARC facility.

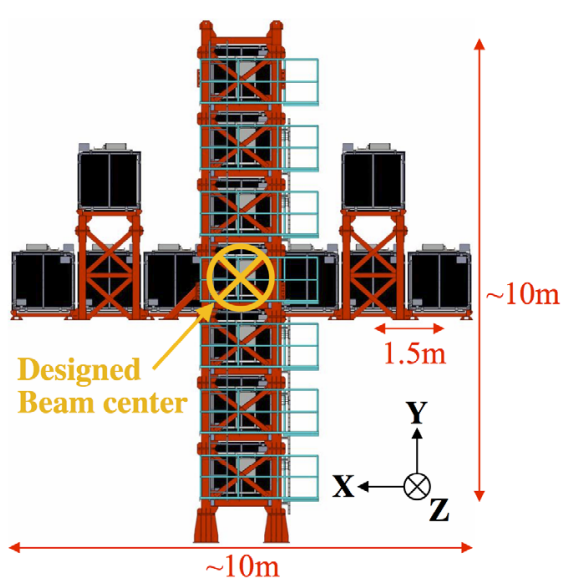

(a) INGRID detector configuration.

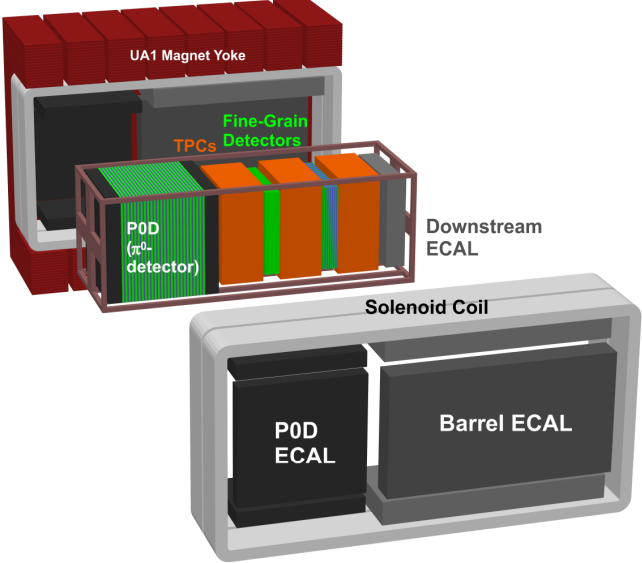

(b) Exploded diagram of ND280 configuration. Beam passes from left to right.

Figure 2: T2K near detectors. 


\subsubsection{INGRID}

INGRID [4] is comprised of 14 individual modular detectors placed in a cross configuration, as shown in Figure 2a, which is primarily designed to monitor the neutrino beam direction and profile. Two additional off-axis modules measure the beam asymmetry. Each INGRID module is built up of planes of iron and scintillator, their high mass, and therefore high interaction rate, allows for the neutrino beam to be monitored at a per beam-spill level.

\subsubsection{ND280}

ND280 is designed to characterise the off-axis beam composition, prior to oscillations having occurred. This requires measuring the unoscillated $v_{\mu}$ spectrum, along with the inherent $v_{e}$ and wrong sign $\left(\bar{v}_{\mu}\right.$ and $\left.\bar{v}_{e}\right)$ beam contamination (for neutrino operation, particles and antiparticles are reversed for antineutrino operation). The ND280 is a complex detector design, see Figure 2b, featuring both carbon and water target regions within fine-grained scintillator detectors. It has a $0.2 \mathrm{~T}$ magnetic field to allow for charge discrimination, momentum measurements and particle identification of charged tracks within time projection chambers.

\subsection{Far Detector, Super-Kamiokande}

The T2K far detector is Super-Kamiokande [5]; located $295 \mathrm{~km}$ from J-PARC it measures the oscillated $v_{\mu}$ and $v_{e}$ spectra. SK is a $50 \mathrm{kT}$ (22.5 kT fiducial volume) water Cherenkov detector, readout by over 11,000 20 inch photo-multiplier tubes. SK is capable of distinguishing between $\mu$ and $e$ interactions, based upon the different Cherenkov ring profiles, with greater than $99 \%$ accuracy.

\subsection{Datasets}

T2K has been operating since 2009 and the results presented here are based on the delivery of $6.57 \times$ $10^{20}$ Protons-On-Target (POT) in neutrino mode operation, and $4.01 \times 10^{20}$ POT in antineutrino mode operation.

\section{Oscillation Analysis Methodology}

The T2K oscillation analysis works by extrapolating the measured, unoscillated, neutrino spectrum at the near detectors, to produce an event rate prediction at SK. The prediction is based on parametrisation of the flux, cross section and detector simulation models. The flux and cross section parameters are initially constrained by external measurements and T2K beamline monitoring, which provide an initial event rate prediction at the ND280. These parameters are then tuned via a fit to ND280 data samples, allowing production of an unoscillated event rate prediction at SK. A further fit is then performed to reconcile the unoscillated prediction with the oscillated measurement at SK, allowing the extraction of the oscillation parameters within the PMNS framework.

\subsection{Flux Model}

FLUKA [6] is used to model the interaction of the protons with the T2K target, which is tuned to external hadron production data and NA61/SHINE [7] measurements of pion and kaon production data on a carbon target. GEANT3 [8] with GCALOR [9] is then used to simulate the subsequent 
particle propagation and decay. INGRID and beam monitors provide additional constraint on the initial unoscillated neutrino flux prediction as shown in Figure 3. Prior to any ND280 constraint the flux uncertainty is about $10 \%$, and the covariance between ND280 and SK allows for this uncertainty to be reduced by the ND280 fit.

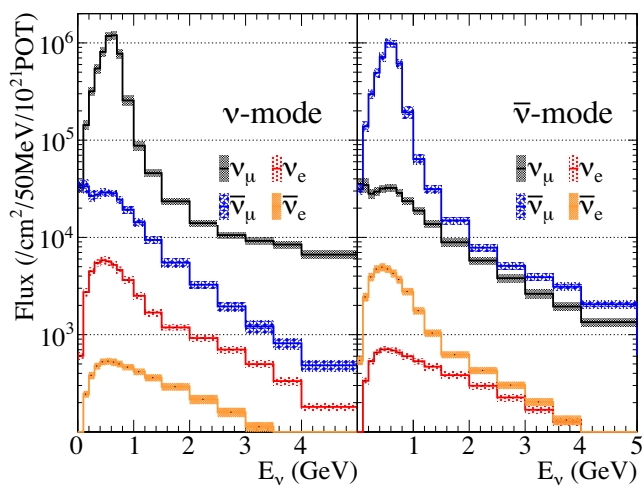

Figure 3. T2K flux prediction at SK in neutrino and antineutrino mode operation, the bands represent the flux uncertainty [10].

\subsection{Cross Section Model}

Neutrino interactions are modelled using NEUT [11], with the oscillation signal extracted from charged-current quasi-elastic (CCQE) interactions. Unfortunately there is significant uncertainty on current measurements of CCQE interaction cross sections, as seen in Figure 4, and non-CCQE interactions can mimic the CCQE signal, for example via pion absorption or multi-nucleon interactions. Therefore it is crucial to include all relevant physics models within the NEUT interaction generator and they are correctly parametrised. T2K tunes the NEUT model parameters to external cross section measurements, such as those from MiniBooNE [12] and MINER $v$ A [13], along with pion-nucleus scattering data to model pion transportation within the nuclear environment.

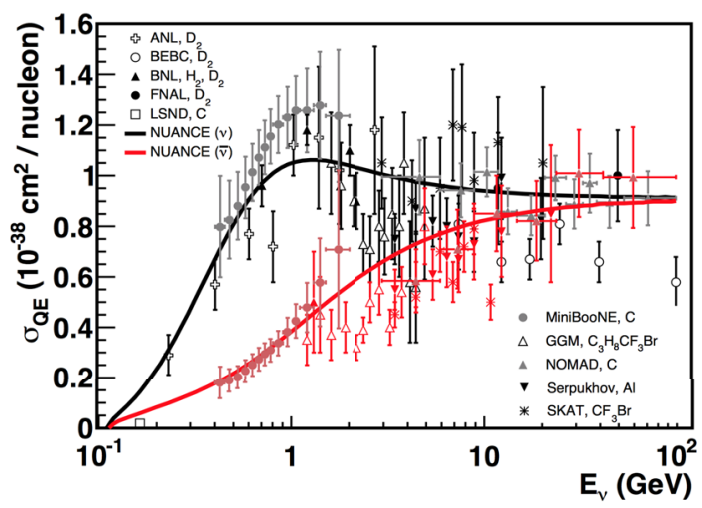

Figure 4. Neutrino (black) and antineutrino (red) CCQE cross section measurements showing significant spread and large uncertainties [1]

\subsection{ND280 Fit}

The T2K flux and cross section models, in combination with the ND280 GEANT4 [14] simulation, allow for an initial prediction of interaction rates in the ND280. Event samples are then selected by 


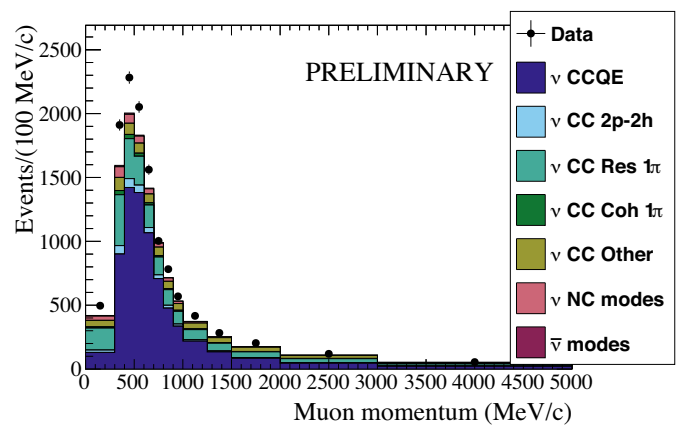

(a) Prefit.

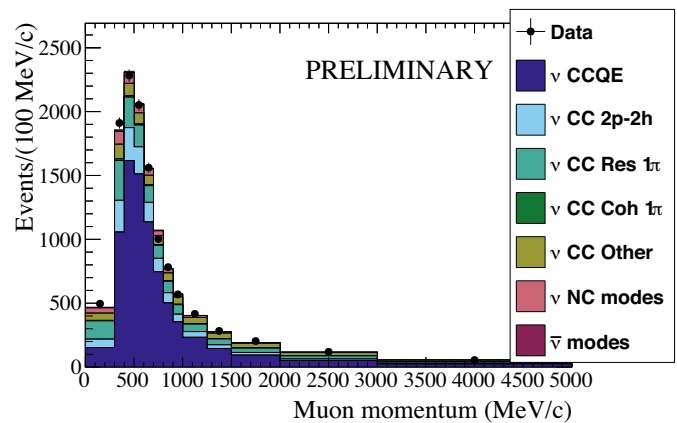

(b) Postfit.

Figure 5: Neutrino beam mode, $0 \pi$ sample, muon momentum distribution pre (left) and post (right) ND280 flux and cross section fit.

the ND280, and these are used to create a new fit to the cross section and flux parameters to better constrain them.

\subsubsection{ND280 Event Samples}

Seven independent event samples are selected for the ND280 fit, three in neutrino beam and four in antineutrino beam mode. All samples require the selection of the highest momentum, muon-like track, and then use charge discrimination to determine whether it came from a neutrino or antineutrino interaction. For neutrino beam data the three samples are partitioned based upon the number of pions observed during the interactions, providing, $0 \pi, 1 \pi$ and $N \pi$ charged-current samples. For the antineutrino beam operation the samples are split based on track multiplicity into 1-Track and $N$ Track charge current samples, but due to the large neutrino contamination within the antineutrino beam, these samples are produced with both a positive and negative muon candidate to give four samples in total.

\subsubsection{Parameter Fit}

All seven samples are used to simultaneously fit the flux and cross section model parameters, taking into account the uncertainties in the near detector measurements. This improves the data - Monte Carlo agreement, as shown in Figure 5, and reduces the total flux and cross section uncertainty, as shown in Figure 6 when the new parameters are applied to the SK prediction.

\subsection{Oscillation Fit}

The combination of the ND280 fit and independent cross section models (those not currently constrained by the ND280 fit) are combined with the GEANT3 SK detector model to produce a final event rate prediction at SK. 


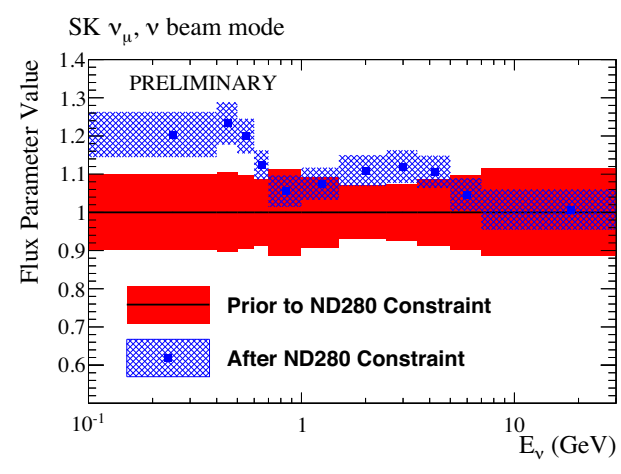

(a) Flux parameters.

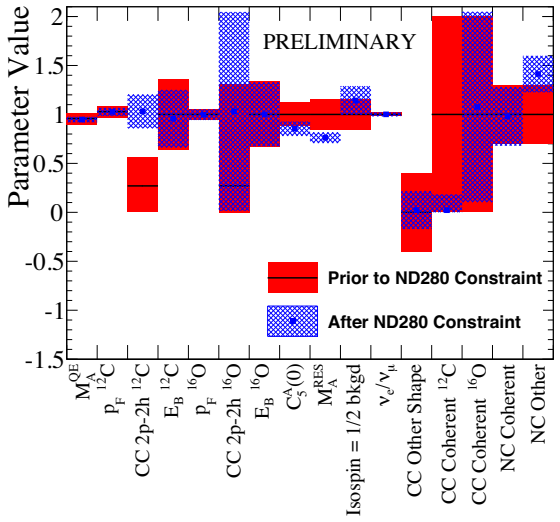

(b) Cross section parameters.

Figure 6: Change in the value and associated uncertainty on the flux (left) and cross section (right) parameters prior to (red) and after (blue) the ND280 fit.

\subsubsection{SK Event Samples}

All event selection requires single, fully contained Cherenkov rings, with a vertex reconstructed within the SK fiducial volume and in temporal coincidence with the J-PARC neutrino beam. These rings are then characterised as $\mu$-like or $e$-like based on a likelihood fit.

If a ring is found to be $\mu$-like it is also required to have a reconstructed momentum greater than $200 \mathrm{MeV} / \mathrm{c}$ (to reject charged pions and misidentified electrons), and that there are less than two decay electrons reconstructed (to reject events with unobserved muons or pions).

For $e$-like rings the additional requirements are that the visible energy is greater than $100 \mathrm{MeV}$ (to remove low energy neutral-current interactions and the decay electrons from unobserved parent particles), there are no decay electrons, the reconstructed neutrino energy is less that $1.25 \mathrm{GeV}$ (as above this threshold the intrinsic $v_{e}$ beam contamination becomes dominant), and that the event is not consistent with the reconstruction of a $\pi^{0}$. The resultant distributions in data and Monte Carlo (with nominal oscillation parameters) are shown in Figure 7.

\subsubsection{Analyses}

To extract the oscillation parameters a likelihood fit is performed between the selected SK events and the predicted event distributions which are varied across the full oscillation parameter phase-space. The solar oscillation parameters to which the experiment is insensitive $\left(\theta_{12}, \Delta m_{12}^{2}\right)$ are either held at, or Gaussian drawn from, the world best fit value, while the remaining oscillation parameters (either all, or a subset of them) fitted depending on the specific analysis being undertaken, as will be described in Section 4. The analyses that will be described are:

- A single fit to the neutrino beam $v_{\mu}$ disappearance sample;

- A joint fit between the neutrino beam $v_{\mu}$ disappearance and $v_{e}$ appearance samples;

- A single fit to the antineutrino beam $\bar{v}_{\mu}$ disappearance sample; and

- A test for consistency with PMNS oscillations on the antineutrino beam $\bar{v}_{e}$ appearance sample. 


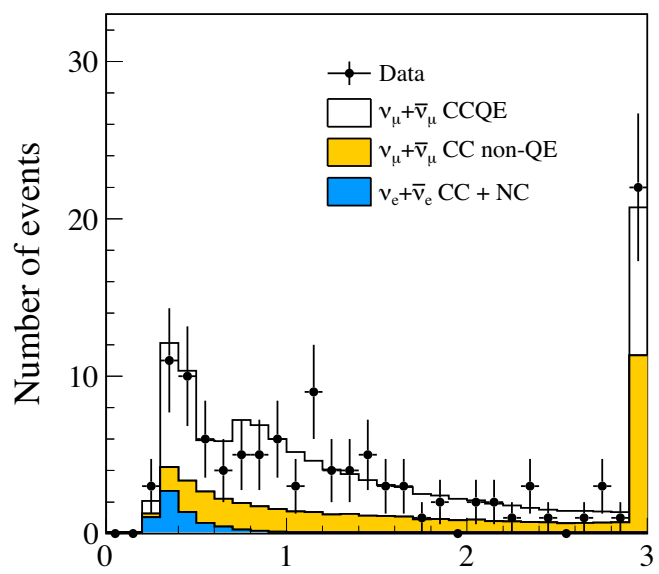

Reconstructed $v$ energy $(\mathrm{GeV})$

(a) $v_{\mu}$ disappearance sample.

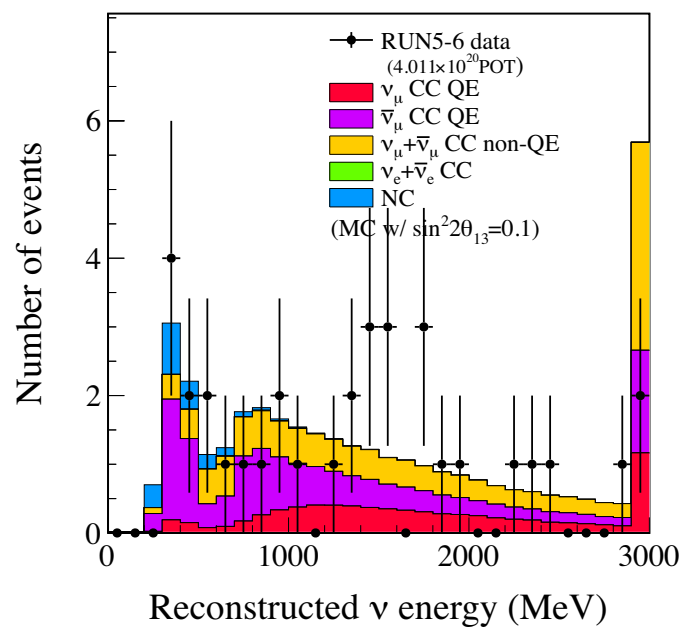

(c) $\bar{v}_{\mu}$ disappearance sample.

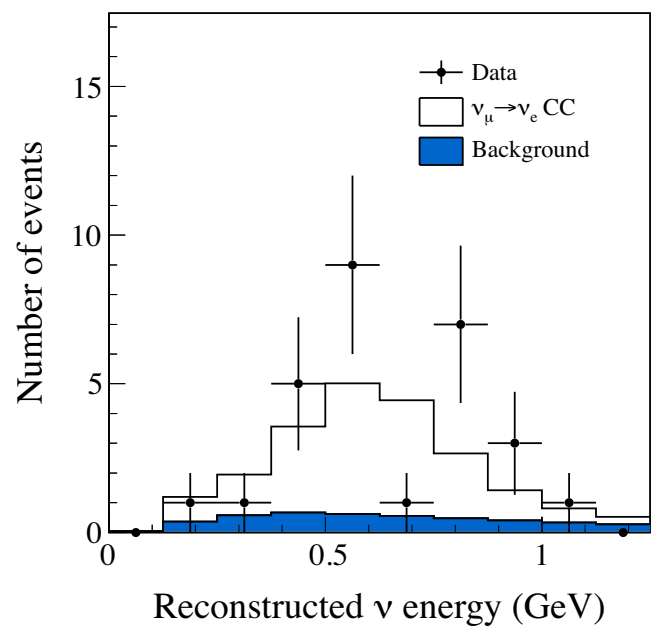

(b) $v_{e}$ appearance sample.

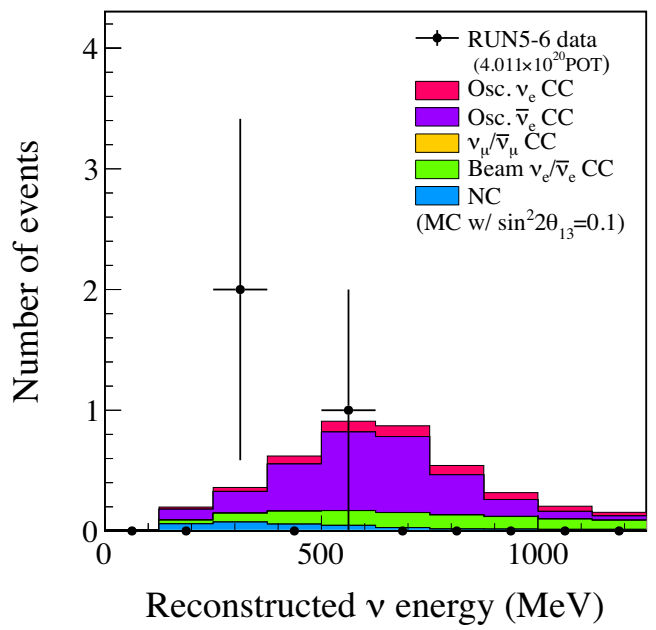

(d) $\bar{v}_{e}$ disappearance sample.

Figure 7: Reconstructed neutrino energy for the four SK event samples.

\section{Results}

\section{1 $v_{\mu}$ Disappearance Fit}

The likelihood fit is performed to the oscillation parameters $\theta_{23}$ and $\Delta m_{32}^{2}\left(\Delta m_{13}^{2}\right)$ assuming the normal (inverted) hierarchy. The parameters $\theta_{12}, \theta_{13}$ and $\Delta m_{12}^{2}$ are Gaussian thrown from the global best fit with errors and the full range of possible values for $\delta$ are sampled $(-\pi \rightarrow+\pi)$. The measurement is sensitive to the $\theta_{23}$ octant, and the results prefer maximal disappearance and the second octant for both the normal and inverted hierarchy assumptions as shown in Figure 8 [15]. 


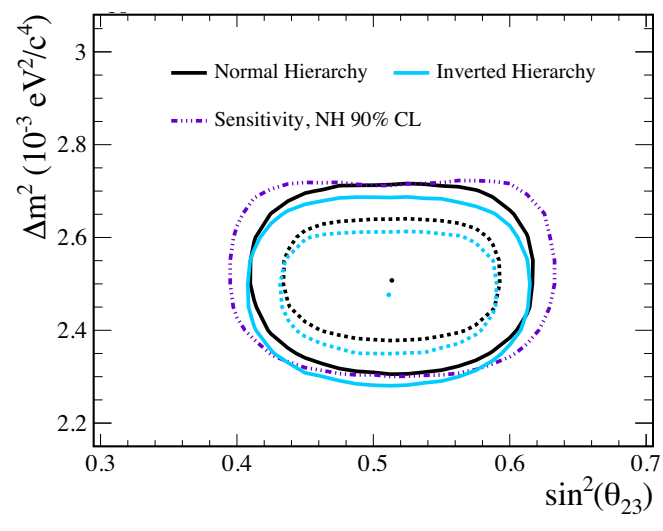

Figure 8. 68\% (dashed) and 90\% (solid) confidence level intervals for $\theta_{23}$ and $\Delta m_{32}^{2}\left(\Delta m_{13}^{2}\right)$ assuming the normal (inverted) hierarchy. The projected $90 \%$ confidence level sensitivity contour is also shown assuming the normal hierarchy.

\section{2 $v_{\mu}$ Disappearance and $v_{e}$ Appearance Joint Fit}

The likelihood fit is performed to the oscillation parameters $\theta_{23}, \theta_{13},\left|\Delta m^{2}\right|$ and $\delta$, where $\left|\Delta m^{2}\right|$ is derived from a simultaneous fit over both mass hierarchies. The parameters $\theta_{12}$ and $\Delta m_{12}^{2}$ are fixed to the global best fit values. The initial fit showed a very weak preference for the inverted hierarchy, the second octant with maximal mixing, and a large value for $\theta_{13}$ but with a significant error envelope that is consistent with the smaller measurements made by reactor experiments. The fit was insensitive to the charge-parity violating phase $\delta$.

T2K is less sensitive to $\theta_{13}$ than the reactor measurements, so this can be used as a constraint within the joint fit by including it as a $\chi^{2}$ penalty term. By including the reactor constraint the fit subsequently favoured the normal hierarchy and the second octant with maximal mixing as shown in Figure 9. The data also begins to show sensitivity to $\delta$, favouring a value around $\delta \approx-\frac{\pi}{2}$, and rejecting $\sim 35 \%(\sim 60 \%)$ of the possible range of $\delta$ at the $90 \%$ confidence level within the normal (inverted) hierarchy, as shown in Figure 10 [15].

\section{3 $\bar{v}_{\mu}$ Disappearance Fit}

Similar to the neutrino mode measurement, this fits $\bar{\theta}_{23}$ and $\Delta \bar{m}_{32}^{2}$ to the $\bar{v}_{\mu}$ contribution of the $\bar{v}_{\mu}$ disappearance sample. The wrong-sign $v_{\mu}$ background contribution to the $\bar{v}_{\mu}$ disappearance sample is also used to simultaneously fit $\theta_{23}$ and $\Delta m_{32}^{2}$ as independent parameters. The parameters $\theta_{12}, \theta_{13}$ and $\Delta m_{12}^{2}$ are fixed to the global best fit values (and are assumed to be the same for neutrinos and antineutrinos), whilst $\delta$ is fixed to -1.55 radians from the previously described joint fit analysis. The hierarchy is assumed to be normal due to the low statistics preventing any sensitivity to this parameter. This measurement weakly prefers the first octant, but is consistent with other experiments and the previous T2K neutrino beam results as shown in Figure 11, and is currently statistics limited [10].

\section{$4.4 \bar{v}_{e}$ Appearance Test}

There are insufficient statistics within the $\bar{v}_{e}$ sample to perform a full analysis fit, instead a discrete parameter $\beta$ is defined to modify the PMNS appearance probability such that

$$
P\left(\bar{v}_{\mu} \rightarrow \bar{v}_{e}\right)=\beta \times P_{\mathrm{PMNS}}\left(\bar{v}_{\mu} \rightarrow \bar{v}_{e}\right) .
$$

Toy experiments are performed with two values of $\beta, \beta=0$ where no oscillations occur, and $\beta=1$ where oscillations occurred in line with the PMNS expectation. Each toy experiment throws the 

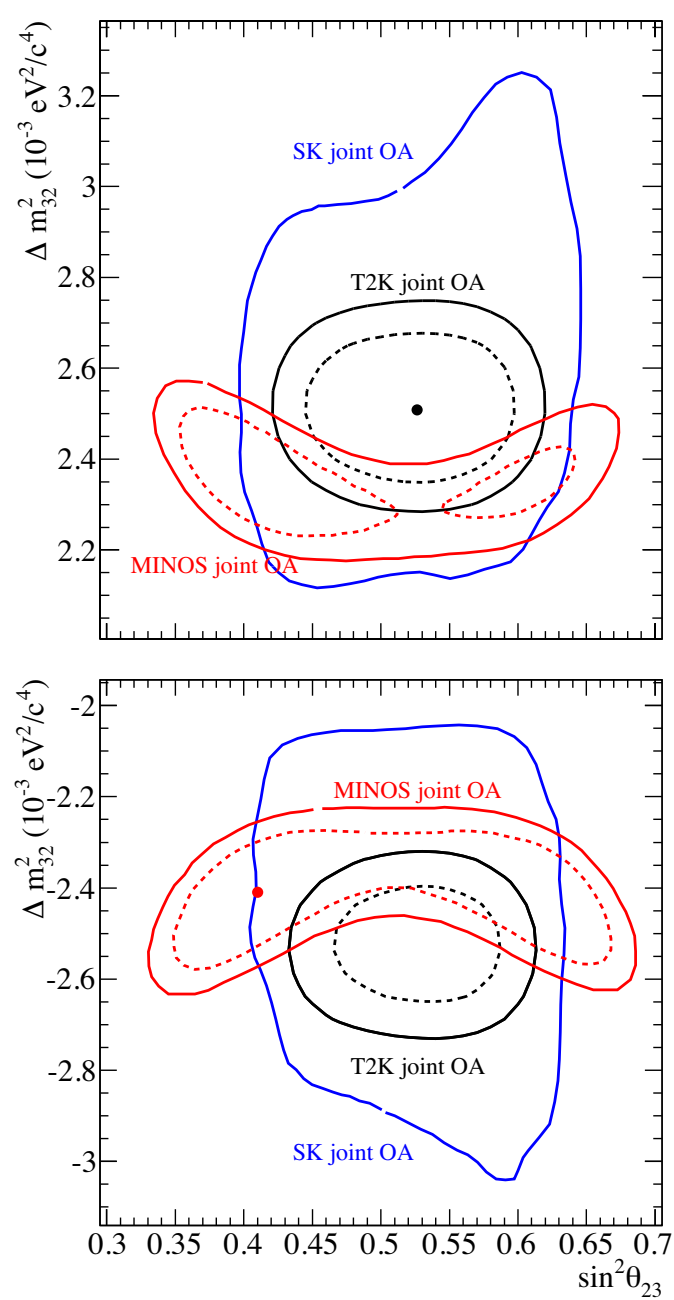

Figure 9. 68\% (dashed) and 90\% (solid) confidence level intervals for $\theta_{23}$ and $\Delta m_{32}^{2}$ showing the distribution across the normal (upper) and inverted (lower) hierarchies. Results from the MINOS [16] and SK [17] experiments are also shown for comparison.

parameters $\theta_{13}, \theta_{23}, \Delta m_{32}^{2}$ and $\delta$ whilst fixing $\theta_{12}$ and $\Delta m_{12}^{2}$ the global best fit values. The distribution in expected number of events from each toy throw is shown in Figure 12 for both $\beta$ hypotheses. As can be seen the average expectation for the no oscillation hypothesis, $\beta=0$, was for $\sim 1.6$ events, and under the PMNS oscillation hypothesis, $\beta=1$, was for $\sim 4$ events. With 3 events recorded in data there is no way to distinguish between these two hypotheses and this will require more data to be taken.

\section{Future Sensitivity}

Subsequent results will utilise a joint fit between all four SK event samples. In doing so T2K will be able to begin to probe $\delta$ at high precision. Figure 13 shows the potential sensitivity of T2K to $\delta$ as a function of $\theta_{13}$ assuming true $\delta=-\frac{\pi}{2}$ and a $50 \%$ neutrino beam and $50 \%$ antineutrino beam mix delivering $7.8 \times 10^{21}$ POT [20]. The systematic uncertainties used are taken from the $2012 \mathrm{~T} 2 \mathrm{~K}$ oscillation analyses and are $\sim 15 \%$, a figure that has already been improved upon within the most 

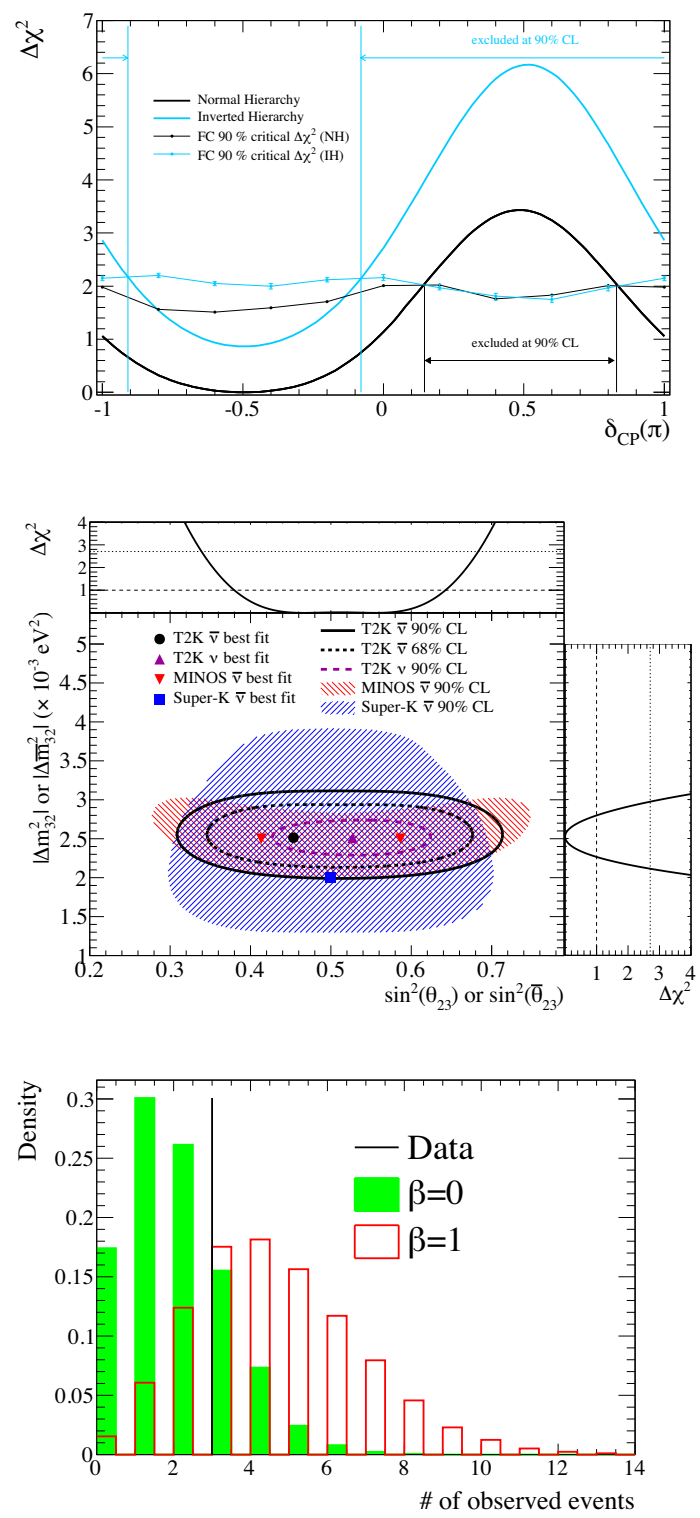

Figure 10. Profiled $\Delta \chi^{2}$ distribution as a function of $\delta$ for the normal (black) and inverted (blue) hierarchy, with the $90 \%$ confidence level exclusion critical values displayed.
Figure 11. 68\% (dashed) and 90\% (solid) confidence level intervals for $\bar{\theta}_{23}$ and $\Delta \bar{m}_{32}^{2}$. Results from the MINOS [18] and SK [19] experiments are also shown for comparison, along with the T2K [15] neutrino beam result.
Figure 12. Distribution for the predicted number of events from the $\beta=0$ and $\beta=1$ toy experiments along with the data result.

recent analyses. Further constraint from the reactor experiments along with measurements from the $\mathrm{NO} v \mathrm{~A}$ experiment [21][22] will further improve upon this future sensitivity.

\section{Conclusion}

With current data, about $\sim 14 \%$ of the planned total, T2K has achieved world leading measurements of the oscillation parameter $\theta_{23}$ and has begun to probe $\delta$. Future updates will include higher statistics, an improved near detector fit (with water target data), full four SK sample joint fit, and the inclusion of additional datasets from the SK charged-current $1 \pi^{ \pm}$samples. T2K is also producing a large number 


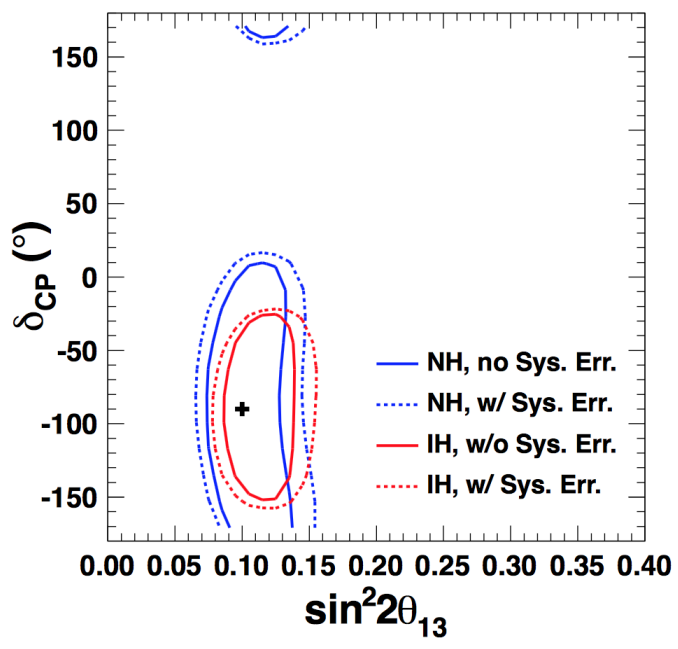

Figure 13. Future sensitivity of T2K to $\delta$ and $\theta_{13}$ displaying $90 \%$ confidence level contours with and without systemic uncertainty for both the normal and inverted hierarchy.

of cross section measurements, and is beginning work on proposed future extensions to the experiment such as T2K-II and Hyper-Kamiokande.

\section{References}

[1] K.A. Olive et al. (Particle Data Group), Chin. Phys. C38, 090001 (2014)

[2] K. Abe et al., Nucl. Instrum. Meth. 659, 106 (2011)

[3] K. Abe et al. (T2K Collaboration), Phys. Rev. D 87, 012001 (2013)

[4] K. Abe et al., Nucl. Instrum. Meth. 694, 211 (2012)

[5] S. Fukuda et al., Nucl. Instrum. Meth. 501, 418 (2003)

[6] A. Ferrari et al., Tech. rep. (2005)

[7] N. Abgrall et al., Nucl. Instrum. Meth. 701, 99 (2013)

[8] R. Brun et al., Tech. rep., CERN DD/EE/84-1 (1987)

[9] C. Zeitnitz, T.A. Gabriel, Nucl. Instrum. Meth. A349, 106 (1994)

[10] K. Abe et al. (T2K Collaboration), Phys. Rev. Lett. 116, 181801 (2016)

[11] Y. Hayato, Acta Phys. Polon. B40, 2477 (2009)

[12] A. Aguilar-Arevalo et al., Nucl. Instrum. Meth. 599, 28 (2009)

[13] L. Aliaga et al., Nucl. Instrum. Meth. 743, 130 (2014)

[14] S. Agostinelli et al., Nucl. Instrum. Meth. 506, 250 (2003)

[15] K. Abe et al. (T2K Collaboration), Phys. Rev. D 91, 072010 (2015)

[16] P. Adamson et al., Phys. Rev. Lett. 112, 191801 (2014)

[17] A. Himmel (The Super-Kamiokande Collaboration), AIP Conf. Proc. 1604, 345 (2014)

[18] P. Adamson et al. (The MINOS Collaboration), Phys. Rev. Lett. 108, 191801 (2012)

[19] K. Abe et al. (Super-Kamiokande Collaboration), Phys. Rev. Lett. 107, 241801 (2011)

[20] K. Abe et al., Prog. Theor. Exp. Phys. 2015, 043 C01 (2015)

[21] P. Adamson et al. (NOvA Collaboration), Phys. Rev. D 93, 051104 (2016)

[22] P. Adamson et al. (NOvA Collaboration), Phys. Rev. Lett. 116, 151806 (2016) 